\title{
Reperfusion, Conditioning and the On-Going Search for the Holy Grail
}

\author{
Robert L Yellon, Rob M Bell
}

Dr Robert L Yellon, Dr Rob M Bell

The Hatter Cardiovascular Institute

University College London

67 Chenies Mews, London

WC1E 6HX, UK

Email: r.yellon@ucl.ac.uk; rob.bell@ucl.ac.uk

Tel: 02034479888

Word Count: 1824

Conflicts of interest: none

Key words: ischaemic conditioning; pre-conditioning; per-conditioning; post-conditioning; reperfusion injury; IPC; RIPC; PPerC; RIPerC; PPost; IPost; RIPost 


\section{Abstract}

Ischaemic conditioning is the phenomenon of protection against reperfusion injury via the application of brief, repeated episodes of non-lethal ischaemia. This review has three aims: 1) to briefly explain the various categories of ischaemic conditioning; 2 ) to explore past clinical trials and their failures; 3 ) to explore the future of clinical trials in the realm of ischaemic conditioning.

\section{Introduction}

Protecting the myocardium against lethal ischaemia necessitates rapid reperfusion. Paradoxically however, reperfusion injures the myocardium, a phenomenon known as "ischaemia-reperfusion injury" (IRI). There is currently no effective clinical intervention for IRI in spite of the fact that it may contribute up to $50 \%$ of final myocardial infarct (MI) size ${ }^{1}$. This review intends to describe past, present, and future clinical trials into ischaemic conditioning, which present one of the few avenues currently being explored clinically to kerb IRI.

\section{The Principles of Ischaemic Conditioning and the Supporting Proof-of-Concept Studies}

Ischaemic Conditioning is a form of protection against IRI produced via brief cycles of non-lethal ischaemia. These cycles may be sub-classified thusly (see also figure 1):

\section{- Pre-conditioning}

- Classical Ischaemic Pre-conditioning (IPC): brief cycles of non-lethal ischaemia induced locally and directly to the myocardium prior to an episode of lethal ischaemia, e.g. aortic clamping. IPC has been shown to reduce post-surgical markers of cardiovascular damage (e.g. ATP2, Troponin $\mathrm{T}^{3}$ ) when performed during coronary artery bypass surgery (CABG). However, due to the inherent embolic risk of such intervention, it has not been carried over to large-scale clinical trials.

- Remote Ischaemic Pre-conditioning (RIPC): brief cycles of non-lethal ischaemia induced at a "remote" limb via blood pressure cuff prior to an episode of lethal 
myocardial ischaemia. The need to apply the conditioning prior to an ischaemic stimulus largely limits its application to the surgical arena where the time of onset of the injurious ischaemia can be readily predicted. Proof-of-concept studies have shown that RIPC has the capacity to reduce post-CABG troponin release in humans. ${ }^{4,5}$ Such an intervention is an attractive prospect due to its non-invasive nature. However, the recent outcome trials, RIPHEART and ERICCA, have been neutral and will be explored later in this review.

\section{- Per-Conditioning}

- Pharmacological Per-conditioning (PPerC): the use of pharmaceutical agents to mimic a conditioning stimulus during an ischaemic insult to protect against subsequent reperfusion injury. Proof-of-concept studies have shown cyclosporine-A $(\mathrm{CsA})^{6}$ and metoprolol ${ }^{7}$ to be associated with a significant reduction in infarct size (measured by late-gadolinium enhancement on cardiac MRI). However, the largescale CSA-based CIRCUS and CYCLE studies have also been neutral and are discussed in the next section ("The Failure of Large-Scale Ischaemic Conditioning Trials").

- Remote Ischaemic Per-conditioning (RIPerC): brief cycles of non-lethal ischaemia induced at a limb via blood pressure cuff during an episode of lethal myocardial ischaemia. The proof-of-concept ERIC-LYSIS ${ }^{8}$ study randomised ST-elevation myocardial infarction (STEMI) patients to receive thrombolysis with or without RIPerC. The RIPerC cohort had a significant reduction in MI size 24 hours postintervention. This was shown by a median $32 \%$ reduction in troponin-T $(P=0.020)$, and $19 \%$ reduction in creatinine kinase $(P=0.026)$ levels. The proof-of-concept $\mathrm{CONDI}^{9}$ trial randomised STEMI patients to receive $\mathrm{PPCl}$ with or without in-ambulance RIPerC. The RIPerC cohort had a significant reduction in all-cause mortality $(P=0.027)$ on follow-up (median $=3.8$ years). The large-scale CONDI2/ERICPPCI studies are 
underway and will be discussed in the final section ("The Future of Clinical Trials in Ischaemic Conditioning").

\section{- Post-conditioning}

- Pharmacological Post-conditioning (PPost): the use of pharmaceutical agents to mimic a conditioning stimulus following onset of reperfusion to reduce subsequent reperfusion injury. The J-WIND study of $569 \mathrm{MI}$ patients ${ }^{10}$ showed that those given an atrial natriuretic peptide (ANP) infusion post-reperfusion had a significantly reduced infarct size of $14.7 \%(P=0.019)$ and a reduced incidence of readmission for heart failure relative to the control group (median follow-up period $=2.7$ years) $(H R=0.267$; $P=0.011)$. This same study showed that nicorandil had no effect on infarct size. In a study of 172 STEMI patients, ${ }^{11}$ exenatide (glucagon-like-peptide-1 analogue) infusion during reperfusion attenuated myocardial infarction, resulting, on average, in a 15\% greater salvage index (i.e. the difference between actual and potential infarct size). In spite of this however, no significant change in clinical outcome between the two cohorts was found.

Classical Ischaemic Post-conditioning (IPost): brief cycles of non-lethal ischaemia applied following angioplasty of the culprit lesion, typically achieved via transient balloon inflation within the culprit vessel. Proof-of-concept studies have produced mixed results ${ }^{12,13}$ and the large-scale DANAMI 3-iPost (data presented at the American College of Cardiology 2016) clinical trial showed no significant difference in clinical outcomes. This trial will be explored in the next section ("The Failure of Large-Scale Ischaemic Conditioning Trials").

- Remote Ischaemic Post-conditioning (RIPost): brief cycles of non-lethal ischaemia induced at a limb via blood pressure cuff following reperfusion therapy. Whilst proofof-concept studies have shown some promise ${ }^{14}$, no large-scale clinical trials have yet been undertaken to explore RIPost. 


\section{The Failure of Large-Scale Ischaemic Conditioning Trials}

Whilst proof-of-concept studies have provided a tantalising view of potential clinical efficacy with various ischaemic conditioning interventions, large-scale clinical trials have so far proved fruitless. This section will explore these failed trials and examine why ischaemic conditioning techniques have yet to fulfil their clinical potential.

- RIPC - The 2015 RIPHeart (“A Multicentre Trial of Remote Ischaemic Preconditioning for Heart Surgery) study ${ }^{15}$ by Meybohm et al was a 1403 patient, multicentre, double-blinded, randomised controlled trial (RCT) exploring RIPC in elective surgical patients requiring cardiac bypass. The primary end-point was a composite of acute renal failure, stroke, non-fatal MI or all-cause death up to 14 days after surgery. Four cycles of true or sham RIPC were given postanaesthetic induction and pre-surgical incision. No significant difference was seen between the primary end-points of either cohort. At approximately the same time, the ERRICA ("The Effect of Remote Ischaemic Conditioning on Clinical Outcomes in Coronary Artery Bypass Graft Surgery") study ${ }^{16}$ by Yellon et al was published. This study was a 1612 patient, multicentre, double-blinded RCT of RIPC in patients undergoing CABG surgery. The primary end-point was the rate of major adverse cardiac and cerebral events 12 months after the intervention. Four cycles of true or sham RIPC were given post-anaesthetic induction and pre-surgical incision. Once again, no significant difference was seen between the primary end-points of either cohort.

Why was it that both of these trials failed to provide positive results? It may be that the injury suffered during CABG surgery is too small for RIPC to provoke a significant protective effect: it is well recognised from the pre-clinical literature that the smaller the primary injury, the smaller the benefit from a conditioning intervention is likely to be. It is also important to consider the other therapies patients undergoing surgical procedures will be exposed to: all patients in RIPHeart and $>90 \%$ of patients in ERICCA were anaesthetised with propofol, an 
agent which might in-fact abolish RIPC-based cardio-protection and an important potential confounder ${ }^{17}$.

- PPerC - The 2015 CIRCUS (“̌yclosporine to ImpRove Clinical outtcome in $\underline{\text { ST}}$-elevation Myocardial Infarction Patients") study ${ }^{18}$ by Cung et al was a 970 patient, multicentre, doubleblinded RCT exploring CSA as a preconditioning agent in patients undergoing PPCl to treat STEMI. The primary end point was a 1- and 3-year composite of heart failure progression, rehospitalisation for heart failure, adverse left ventricular modelling, and all-cause death. Prior to $\mathrm{PPCl}$, patients were randomised to a CsA or placebo infusion. No significant difference in the primary end point was seen at 1 year: a neutral outcome. This could be due to the absence of data for LV end-diastolic volume in $17 \%$ of patients. This absent data, combined with the high incidence of adverse modelling in both cohorts, could have made it difficult to detect a significant difference in the other components of the primary end point composite. The 2016 CYCLE ("CYCLosporinE A in Reperfused Acute Myocardial Infarction") study ${ }^{19}$ by Ottani et al was a 410 patient, multicentre, Prospective/Randomised/Open-label/BlindedEndpoint (PROBE) trial which also explored CSA as a perconditioning agent in patients undergoing PPCI to treat STEMI. The primary end point was the incidence of $\geq 70 \%$ ST-segment resolution $60 \mathrm{~min}$ after Thrombolysis in Myocardial Infarction (TIMI) flow grade 3. Prior to $\mathrm{PPCl}$, patients were randomised to a CsA or placebo injection. Once again, no significant difference between the cohorts was detected. As such, the future use of CsA as a perconditioning agent appears doubtful.

- IPost - DANAMI 3-iPost ("DANish Study of Optimal Acute Treatment of Patients with STsegment Elevation Myocardial Infarction) is a treatment subgroup of a 2016 trial programme comparing multiple treatment strategies for STEMI. The iPost arm of the trial was a 1234 patient, multicentre, PROBE trial comparing standard PCI to PCI plus IPost. The primary end point was a composite of hospitalisation for heart failure and all-cause death within 2 years. Patients were randomised to $\mathrm{PCl}$, or $\mathrm{PCl}$ plus four 30 -second balloon inflations within the 
reperfused coronary artery following initial reperfusion. No significant difference was detected between the two cohorts (data presented at the American College of Cardiology, 2016). The duration of ischaemia is critical: in the pre-clinical literature, there is a clear "therapeutic window": ischaemic post-conditioning is ineffective where the index ischaemia duration is either very short or very long ${ }^{20}$ and it would seem that in clinical trials, the therapeutic window is typically in the order of 1-4 hours from symptom onset to revascularisation. Whether or not ischaemic time played a role in the neutral DANAMI 3-iPost study will require further analysis upon official publication.

\section{The Future of Clinical Trials in Ischaemic Conditioning}

A number of large outcome trials exploring ischaemic conditioning are currently underway and may yet still provide evidence for the introduction of conditioning techniques into clinical practice.

\section{RIPerC in Primary PCI}

The CONDI 2 (Effect of RIC on Clinical Outcomes in STEMI Patients Undergoing PPCI) and ERIC-PPCI (Effect of Remote Ischaemic Conditioning on Clinical Outcomes in STEMI Patients Undergoing PPCI) are a pair of collaborative studies investigating the use of RIPerC in STEMI patients. ${ }^{21}$ The studies are both multicentre, multinational, double-blinded RCTs with 2300 patients and 2000 patients respectively. Both trials share identical primary endpoints of hospitalisation for heart failure or cardiovascular mortality at 1 year. In these trials, STEMI patients will be randomised to PPCI with or without RIPerC. The RIPerC will be delivered either in-ambulance or on arrival to the PPCI centre depending on average national transit time. The results of these trials are hoped to be released in approximately 18 months.

\section{RIPerC in Thrombolysis}

Though the ERIC-lysis ${ }^{8}$ study provided evidence for clinically applied RIPerC, no large-scale trials have explored RIPerC in STEMI patients treated with thrombolysis. Whilst thrombolysis as an intervention 
has largely been superseded by $\mathrm{pPCl}$ in the developed world, in the developing world thrombolysis is remains an important first-line therapy for STEMI. Large-scale human trials are still needed to provide evidence for a cost-neutral, life-saving therapeutic adjunct to thrombolysis.

\section{PPerC in pPCl}

Though the aforementioned CIRCUS $^{18}$ study of PPerC was neutral at 1 year, the trial has not yet completed follow-up. It may yet show an effect of CsA at the 3-year end-point and as such, full conclusions may not currently be drawn. It is also worth noting that CsA is not the sole pharmacological agent: there are multiple potential pharmacological targets within the increasingly well characterised cell-death pathway that may yet yield an efficacious PPerC option. Indeed, repurposing existing cardiovascular drugs may yield significant benefit to improve cardiovascular outcomes following an acute myocardial infarction.

\section{Conclusion}

Reperfusion injury plays a significant role in the evolution of the final myocardial infarct size yet physicians have no effective tools with which to combat the phenomenon. Recent small-scale human trials exploring the variety of ischaemic-conditioning modalities have shown some promise. If largescale trials can provide the evidence, reperfusion injury may yet become amenable to treatment. In remote conditioning exists a potentially cheap and non-invasive method of therapy, but as with pharmacological conditioning, the holy grail of an effective clinical intervention against IRI has yet to be realised. However, the search for an effective adjunct to reperfusion, if successful, will be wellrewarded by further reductions in cardiovascular mortality and morbidity and the consequent reduction upon the burgeoning socioeconomic healthcare burden worldwide.

\section{Figure legends}


Figure 1: Cartoon summarising the various forms of conditioning - ischaemic, remote and pharmacological - and the terminology relative to the onset of injurious ischaemia and subsequent reperfusion. Modalities applied prior to injurious ischaemia are termed "preconditioning". A modality applied during the ischaemic injury is "perconditioning". Any modality applied following the restoration of blood flow and reperfusion is regarded as "postconditioning".

\section{References}

1. Yellon DM, Hausenloy DJ. Myocardial reperfusion injury. N Engl J Med. 2007;357(11):1121-35.

2. Yellon DM, Alkhulaifi AM, Pugsley WB. Preconditioning the human myocardium. Lancet. $1993 ; 342(8866): 276-7$.

3. Jenkins DP, Pugsley WB, Alkhulaifi AM, Kemp M, Hooper J, Yellon DM. Ischaemic preconditioning reduces troponin $\mathrm{T}$ release in patients undergoing coronary artery bypass surgery. Heart. $1997 ; 77(4): 314-8$.

4. Hausenloy DJ, Mwamure PK, Venugopal V, et al. Effect of remote ischaemic preconditioning on myocardial injury in patients undergoing coronary artery bypass graft surgery: a randomised controlled trial. Lancet. 2007;370(9587):575-9.

5. Venugopal V, Hausenloy DJ, Ludman A, et al. Remote ischaemic preconditioning reduces myocardial injury in patients undergoing cardiac surgery with cold-blood cardioplegia: a randomised controlled trial. Heart. 2009;95(19):1567-71.

6. Piot $\mathrm{C}$, Croisille $\mathrm{P}$, Staat $\mathrm{P}$, et al. Effect of cyclosporine on reperfusion injury in acute myocardial infarction. N Engl J Med. 2008;359(5):473-81.

7. Ibanez B, Macaya C, Sánchez-brunete V, et al. Effect of early metoprolol on infarct size in STsegment-elevation myocardial infarction patients undergoing primary percutaneous coronary 
intervention: the Effect of Metoprolol in Cardioprotection During an Acute Myocardial Infarction (METOCARD-CNIC) trial. Circulation. 2013;128(14):1495-503.

8. Yellon DM, Ackbarkhan AK, Balgobin V, et al. Remote Ischemic Conditioning Reduces Myocardial Infarct Size in STEMI Patients Treated by Thrombolysis. J Am Coll Cardiol. 2015;65(25):2764-5.

9. Sloth AD, Schmidt MR, Munk K, et al. Improved long-term clinical outcomes in patients with STelevation myocardial infarction undergoing remote ischaemic conditioning as an adjunct to primary percutaneous coronary intervention. Eur HSeart J. 2014;35(3):168-75.

10. Kitakaze M, Asakura M, Kim J, et al. Human atrial natriuretic peptide and nicorandil as adjuncts to reperfusion treatment for acute myocardial infarction (J-WIND): two randomised trials. Lancet. 2007;370(9597):1483-93.

11. Lønborg J, Vejlstrup N, Kelbæk H, et al. Exenatide reduces reperfusion injury in patients with STsegment elevation myocardial infarction. Eur Heart J. 2012;33(12):1491-9.

12. Lønborg J, Kelbaek H, Vejlstrup N, et al. Cardioprotective effects of ischemic postconditioning in patients treated with primary percutaneous coronary intervention, evaluated by magnetic resonance. Circ Cardiovasc Interv. 2010;3(1):34-41.

13. Freixa X, Bellera N, Ortiz-pérez JT, et al. Ischaemic postconditioning revisited: lack of effects on infarct size following primary percutaneous coronary intervention. Eur Heart J. 2012;33(1):10312.

14. Crimi G, Pica S, Raineri C, et al. Remote ischemic post-conditioning of the lower limb during primary percutaneous coronary intervention safely reduces enzymatic infarct size in anterior myocardial infarction: a randomized controlled trial. JACC Cardiovasc Interv. 2013;6(10):1055-63.

15. Meybohm P, Bein B, Brosteanu O, et al. A Multicenter Trial of Remote Ischemic Preconditioning for Heart Surgery. N Engl J Med. 2015;373(15):1397-407.

16. Hausenloy DJ, Candilio L, Evans R, et al. Remote Ischemic Preconditioning and Outcomes of Cardiac Surgery. N Engl J Med. 2015;373(15):1408-17. 
17. Kottenberg E, Thielmann M, Bergmann L, et al. Protection by remote ischemic preconditioning during coronary artery bypass graft surgery with isoflurane but not propofol - a clinical trial. Acta Anaesthesiol Scand. 2012;56(1):30-8.

18. Cung TT, Morel O, Cayla G, et al. Cyclosporine before PCl in Patients with Acute Myocardial Infarction. N Engl J Med. 2015;373(11):1021-31.

19. Ottani F, Latini R, Staszewsky L, et al. Cyclosporine A in Reperfused Myocardial Infarction: The Multicenter, Controlled, Open-Label CYCLE Trial. J Am Coll Cardiol. 2016;67(4):365-74.

20. Manintveld OC, Te lintel hekkert M, Van den bos EJ, et al. Cardiac effects of postconditioning depend critically on the duration of index ischemia. Am J Physiol Heart Circ Physiol. 2007;292(3):H1551-60.

21. Hausenloy DJ, Kharbanda R, Rahbek schmidt M, et al. Effect of remote ischaemic conditioning on clinical outcomes in patients presenting with an ST-segment elevation myocardial infarction undergoing primary percutaneous coronary intervention. Eur Heart J. 2015;36(29):1846-8. 


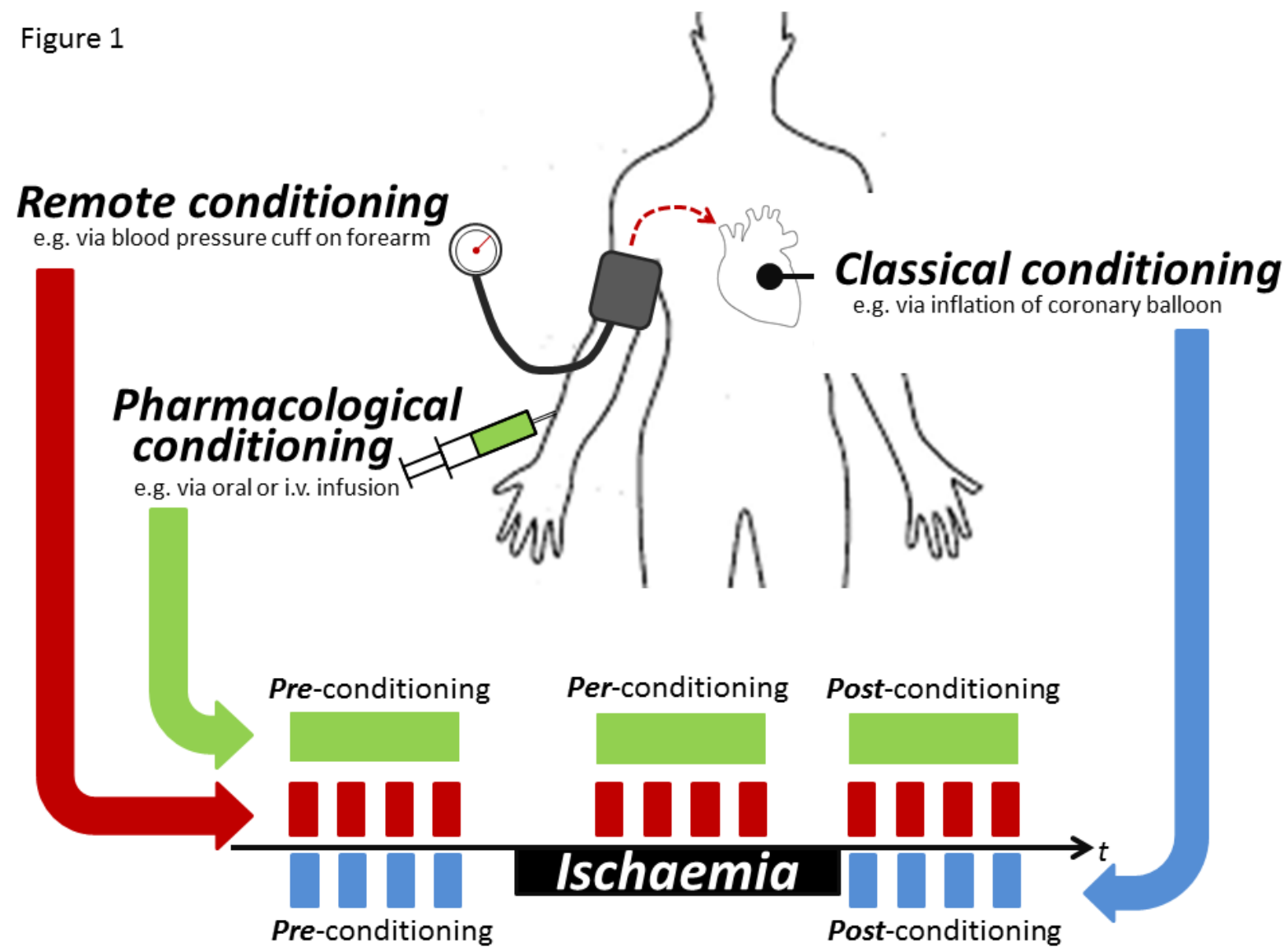

